\title{
Potential Mobilization of Platinum-Group Elements by Siderophores in Surface Environments
}

\author{
Susan R. Dahlheimer, Clive R. Neal*, Jeremy B. Fein
}

This 2-page document includes a detailed description of the experimental methods and Figure S.1 depicting the chemical structure of aHA. 


\section{SUPPORTING INFORMATION}

Sample Preparation and Analysis. Samples in the 12-mL Teflon beakers were acidified with $2 \mathrm{~mL}$ of $12 \mathrm{M} \mathrm{HCl}$ and placed on a hotplate $\left(\right.$ set at $150^{\circ} \mathrm{C}$ ) inside a HEPA-filtered laminar flow fume hood until dry. Two $\mathrm{mL}$ of $0.6 \mathrm{M} \mathrm{HCl}$ was added to the beakers, which were capped, and placed on a hot plate (at $100^{\circ} \mathrm{C}$ ) for 1 hour to ensure complete dissolution of the residue. After cooling the samples were transferred to test tubes and brought up to $3.5 \mathrm{~mL}$ with $0.6 \mathrm{M} \mathrm{HCl}$. The samples were then diluted by a factor of 10 with $0.6 \mathrm{M} \mathrm{HCl}$ to minimize total dissolved solids and internal standards of In (for Pd and $\mathrm{Rh}$ ) and $\mathrm{Tl}$ (for $\mathrm{Pt}$ ) were added for a final concentration of $1 \mathrm{ppb}$. All volumes were recorded gravimetrically and sample preparation took place in a class 100 clean lab. Jewelry was removed during sample handling to avoid potential contamination.

Analyses were performed using the high resolution Thermo Finnigan Element 2 Inductively Coupled Plasma Mass Spectrometer (ICP-MS). A Teflon spray chamber was used to minimize memory effects and $\mathrm{Al}$ cones were found to reduce the interferences on transition metals, which could then interfere on the PGEs. The Teflon sample introduction system implemented a self-aspirating sample flow with cross flow nebulization and used a quartz torch. A two stage washing protocol of $5 \% \mathrm{HCl}$ effectively rinsed the system. The ICP-MS was calibrated using external, matrix-matched calibration standards ranging from $0 \mathrm{ppb}$ to $75 \mathrm{ppb}$. Samples were analyzed ten times in low and medium resolution for all isotopes of each PGE and internal standard. Analysis of all isotopes of Pt and Pd combined with the relatively simple sample matrix, confirmed that significant interferences were not present. Data reduction was performed based on the methods described in [21].

DFO-B Breakdown. Due to the length of these experiments, we hypothesized that DFO-B could be breaking down. Select samples were sacrificed to assess DFO-B breakdown by measuring the UV-vis absorbance of the Fe-DFO-B complex. This was accomplished by adding $20 \mu \mathrm{L}$ of $0.2 \mathrm{~N} \mathrm{HCl}$ to $3 \mathrm{~mL}$ of sample and then adding $20 \mu \mathrm{L}$ of $1.0 \mathrm{M} \mathrm{FeCl}_{3}$. The samples were allowed to react for a few minutes and placed in the UV-vis (Varian Cary 300 Bio UV-Visible Spectrophotometer) for absorbance scans from 400 to $650 \mathrm{~nm}$. 
UV-vis absorbance scans of samples collected from the PdO experiment at 177-214 days and treated with $\mathrm{FeCl}_{3}$ had Fe-DFO-B absorbance peaks located in the range of 474-479 $\mathrm{nm}$. This is slightly higher than the normal range for Fe-DFO-B absorbance peaks (462-475 nm). This slight shift in peak location could be evidence of DFO-B breakdown or more likely is due to the presence of a Pd-DFO-B complex as a series of standards containing fresh DFO-B, soluble Pd (PdCl Custom Grade Standards, Inorganic Ventures, Inc), and 1.0 $\mathrm{M} \mathrm{FeCl}_{3}$ resulted in a peak locations similar to the samples (474-477 $\mathrm{nm}$ ). However, absorbance scans of the $\mathrm{pH}=10$ stock DFO-B solution (used to replace the $3.5 \mathrm{~mL}$ samples) after 195 days, which was never in contact with $\mathrm{Pd}$, resulted in an absorbance peak at $495 \mathrm{~nm}$. This is evidence that suggests high $\mathrm{pH}$ causes the trihydroxamate siderophore to break into three monohydroxamate molecules similar to the siderophore-like ligand acetohydroxamic acid (aHA) (Figure S1). The Fe-aHA absorbance peak is located between 491-500 nm. Although aHA can also complex with metals, it has a lower stability constant for Fe(III) than does DFO-B [34] and could be influencing the steady state $\mathrm{Pd}$ concentration of the $\mathrm{pH}=10 \mathrm{PdO}$ experiment.

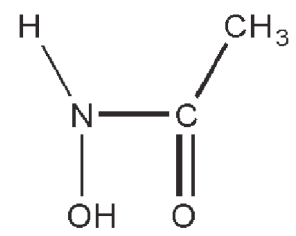

Figure S1. Molecular structure of acetohydroxamic acid (aHA). Note its similarities to the DFO-B hydroxamate groups. 